\title{
Measurement results in a Proficiency Testing programme need to be evaluated against independent criteria
}

\author{
Paul De Bièvre ${ }^{1}$
}

Published online: 15 March 2016

(c) Springer-Verlag Berlin Heidelberg 2016

There exist many interlaboratory comparisons (ILCs). Frequently, they are used to detect, study, and compensate for (un)known effects affecting a measurement result. Another frequent use of ILCs is to evaluate measurement capability. However, an ILC can only be used for one intended use at a time. For the purpose of this Column, we define a PT (Proficiency Testing) programme as an interlaboratory comparison study used for the sole and exclusive purpose of evaluating the measurement capability (proficiency) of one or more participating laboratories.

In a previous Column [1], the declaration by participating laboratories of their measurement results (defined in VIM entry 2.9 [2]) in a Proficiency Testing (PT) programme was discussed. For a definition of measurement capability, see entry 7.3-1 in the IUPAC study referred to in [3]. The usefulness of comparative graphs as easy means to evaluate this measurement capability was stressed. However, a measurement result must comprise its measurement uncertainty in order to be a valid result (see definition in VIM entry 2.9). Sadly enough, that is not done in many cases. Only the measured values are given and compared. Measurement results which do not contain a statement of measurement uncertainty "cannot be compared", as explicitly stated in section 0.1 in [4], yet being "comparable" is "a worldwide need" for measurement

Disclaimer: The author is a member of the Joint Committee on Guides for Metrology (JCGM), Working Group 2 (VIM). The opinions expressed in this Column do not necessarily represent the view of the Working Group or of ACQUAL.

Paul De Bièvre

paul.de.bievre@skynet.be

$1 \quad$ Kasterlee, Belgium results as specified in section 0.3 in [4]. In addition, the metrological traceability (defined in VIM entry 2.41 in [2]) of a result must be indicated. An absence of these two characteristics casts a twofold doubt on such incomplete pictures and, therefore, onto the very validity of any conclusion of the programme. Measurement uncertainty and metrological traceability are intrinsic characteristics of any measurement result and hence also of any participant's measurement result in a PT programme. They are conditions for the very metrological comparability of such results (defined in VIM entry 2.46 [2]). In addition, this metrological traceability must be established to the same reference (defined in VIM entry 2.41 NOTE 1 [2]).

That logically leads to the question against which criterion this evaluation must be performed.

A possible answer is: through the assignment to the programme by the organizer, of a "reference value" (short for "reference quantity value") with a clearly demonstrated metrological traceability. The definition of "reference value" is given in VIM entry 5.18 and its NOTE 2 [2]. Several reference values are possible (VIM entry 5.18 and its NOTE 2 in [2], as made available from:

a. a material, e.g. a certified reference material (CRM),

b. a device, e.g. a stabilized laser,

c. a reference measurement procedure, and

d. a comparison of measurement standards.

One of these, CRM, is defined in entry 5.14 in [2], and another one, "reference measurement procedure", is defined in entry 2.7 in [2].

Let us take a closer look at these definitions. In entry 5.14, a CRM is mentioned as a possible reference. However, any CRM is to be excluded as test sample for a PT programme since its value is for sale and has been certified 
(by definition) and publicly available as such in a certificate and/or certification report. It is, hence, unsuitable as an "unknown" for the participants. In entry 2.7 , the possibility of a reference measurement procedure is created to use a reference value produced by a measurement laboratory, chosen for its metrological competence, and being "external" to the PT programme (i.e. not being one of the participants). In that entry, it is stated that a reference measurement procedure must be "accepted". Acceptance always implies a decision. Thus, it creates the possibility for the PT programme organizer to decide on a reference value based on metrological evidence originating fully from outside the PT programme. This value can be kept "unknown" to the participants until after these submitted their results. That is a value which is independent of the participants' measurement capability (proficiency) because it comes from outside, i.e. it is of "external origin". A decision on the reference value should come, for example, from an NMI-DI (Designated Institute), also called "Reference Laboratory" (RL), designated on the basis of its demonstrated (and accepted) competence. In some contrast to ISO 17043 [5], the suspicion is thereby avoided of making the reference value somehow dependent of the participants' measurement capability, thus opening the way to "circular thinking" contrary to the very intended use of PT programmes for assessing (independently) the measurement capability. [Of course, such an independent reference value must remain unknown to the participants until all participants' values have been collected for evaluation.]

Working on the basis of a designated and demonstrated competence, as well as providing a "reference value" with "external" origin, has the following advantages:

- Evaluation of measurement capability (proficiency) of participants' results in a PT programme is done by comparing their declared value (located somewhere in a stated uncertainty interval) against a value which is not influenced in any way by any of the participants' declared value. It is important that both values be equipped with their measurement uncertainties since all values within a stated uncertainty interval could be the "true" (but unknown) value (see section D.5.2.1 in [4]).

- A comparative graph of the results to which a "reference value" is assigned constitutes an easy tool-even for observers other than analysts-of the measurement capability (proficiency) of participants.

- Solving the problem of removing "outliers" from the graph: they must simply not be removed because they are results of participants which participate to be evaluated for their measurement capability (proficiency).

- Elimination of the calculation of an "average" or "consensus value" which delivers a variable rather than a fixed, hence stable reference value; that avoids the suspicion of arbitrariness in the conclusions from the evaluation.

- An "external" criterion for evaluation of measurement capability (proficiency) obviates the need for having a minimum number of laboratories to calculate an "average" or "consensus" (which is based anyway on the fallacy that the larger the number of measurement results for a given measurand, the closer the average comes to the "true value" for that measurand where all the values within the uncertainty interval could be the true value-for more on "uncertainty" and "true value": see section D.5.2 in [4]).

- In case a "better" laboratory is emerging after a few years of participation in repeated PT programmes, it can be designated by agreement to be a Reference Laboratory for a foreseeable, but limited, future.

Recalling the title of this Column "Results of Proficiency Testing programmes need to be evaluated against independent criteria", it is easy to see that the evaluation of the measurement capability (proficiency) should not be performed against a reference value calculated in any way from the results of participants to the PT programme. It should be a comparison of participants' results against each other's and against a "reference value" originating from a source which is "external" to the PT programme concerned. That enables drafting pictures of the measurement capability of participating laboratories against a stablebecause independently defined-reference. Easy-to-read pictures are easy to interpret for non-technical readers (such as regulatory or legal authorities). It would be helpful if the next revision of ISO 17043 [5] would take this into account.

Maybe there still are other "hidden structures behind the things we observe" which contribute in part to the inconsistencies which we often see in the pictured results of a number of PT schemes.

Identification of these is welcome, preferably as a contribution to the Discussion Forum of this Journal.

\section{References}

1. De Bièvre $P$ (2016) Where do we find the graphs from PT programmes showing measurement performance? Accred Qual Assur 21:87-88. doi:10.1007/s00769-016-1191-2

2. BIPM, IEC, IFCC, ILAC, IUPAC, IUPAP, ISO, OIML (2008), The international vocabulary of metrology-basic and general concepts and associated terms (VIM), edn 3, Joint Committee on Guides for Metrology, JCGM 200:2008/2012 at http://www.bipm. org/vim

3. De Bièvre P, Fajgelj A, Dybkaer R, Hibbert B (2011) Metrological traceability of measurement results in chemistry: concepts and implementation,IUPAC Technical Report. Pure Appl Chem 83:1873-1935 
4. BIPM, IEC, IFCC, ILAC, IUPAC, IUPAP, ISO, OIML (1993/ 1995) Guide to the expression of uncertainty in measurement (GUM), JCGM 100:2008. www.bipm.org/en/publications/guides/ gum.html
5. ISO/IEC 17043:2010 Conformity assessment-General requirements for proficiency testing, International Organization for Standardization, Geneva 